\title{
SEA Subsystem Attribute Correction Based on Stiffness Multipliers
}

\author{
Jie Gao ${ }^{1, *}$ and Yong Yang ${ }^{2}$ \\ ${ }^{1}$ College of Physics and Information Technology, Shaanxi Normal University, Shaanxi, Shanxi,Xian,710119, P.R. China \\ ${ }^{2}$ T\&S Technologies Co. Ltd, Beijing, 100083, P.R. China \\ *Corresponding author
}

\begin{abstract}
This study introduces the principles and application procedures of the stiffness multiplier method used to correct the statistical energy analysis (SEA) subsystem attributes. Using the cockpit floor of a certain type of fighter aircraft as an example, this study combines the finite element method and stiffness multiplier correction technique to modify the modal density of the SEA model through theoretical derivation and numerical simulation analysis. Results of the numerical simulation show that the SEA subsystem modified with the stiffness multiplier method can perfectly reflect the dynamic characteristics of complex stiffened plates. Moreover, the results of the calculation are identical to those obtained with the finite element method. The stiffness multiplier method is found to be applicable to the construction of the complex structures of the SEA subsystem model.
\end{abstract}

Keywords-SEA system correction; stiffness multiplier; modal number; radiation efficiency.

\section{INTRODUCTION}

As an effective prediction technique for medium-high frequency responses, statistical energy analysis (SEA) is widely used in the aerospace and auto industry[1-3]. SEA divides complex systems into different modal groups. In cases of statistical significance, SEA disassembles large systems into several independent subsystems that are easy to analyze instead of precisely determining each modal response. In the initial engineering design stage of SEA applications, the subsystems constituted by modal groups must be defined. The SEA model established in this way can clearly express the characteristics of input, storage, loss, and transmission of vibration energy. The attribute parameters of subsystems can also be calculated accurately to achieve precise dynamic prediction results. However, substructures in practical applications often have complex geometrical and physical properties, and no related attribute parameter is available for reference[4]. Moreover, experiments for testing the attribute parameters of substructures are often time consuming and are thus not widely used in engineering practice. This limitation emphasizes the need to develop a fast and effective method that could predict the attribute parameters of subsystems with complex structures.

In predicting the attribute parameters of subsystems with complex structures, dynamic response (frequency and space average) is usually controlled by a series of "total attributes" even when structures are extremely complex from the physical aspect[5]. The energy storage capacity of structures is controlled by the frequency band within the localized mode, whereas the energy transfer is determined by local attributes near the structural connection (such as local impedance at the structural connection position, transfer loss, and radiation efficiency to the shell structure and acoustic coupling). Therefore, with SEA structures having the same energy storage capacity and transmission properties, accurate SEA subsystems can be established to represent complex structures. This approach establishes a simplified SEA model for complex structures and guarantees accuracy and computational efficiency (main physical parameters that affect structural response).

Over the past decades, several studies have focused on estimating the parameters of SEA subsystems. V. Cotoni and R. Langley estimated the SEA parameters of a periodic structure and presented an analysis theory for periodic structure vibration [6]. Taner Onsay and Anab Akanda et al. developed an estimation method for SEA parameters based on equivalent stiffness [7] and adopted fine finite elements to estimate modal density so as to achieve equivalence in stiffness with a "stiffness multiplier." B. R. Mace and P. J. Shorter adopted the method of energy finite elements to study the coupling relationship between subsystems[8]. On the basis of the work of Taner Onsay and Anab Akanda et al., the present study analyzes the correction of SEA attributes with the stiffness multiplier method and conducts numerical verification using practical structures.

\section{SEA CORRECTION BASED ON STIFFNESS MULTIPLIER}

The main theory of the SEA method is based on the power flow balance equation, with which the energy flow relationship among subsystems can be solved[9]. The power balance relationship among $N$ subsystems is

$$
\omega\left[\begin{array}{cccc}
\left(\eta_{1}+\sum_{i \neq 1} \eta_{i j}\right) N_{1} & -\eta_{21} N_{2} & \ldots & -\eta_{k 1} N_{k} \\
-\eta_{22} N_{1} & \left(\eta_{2}+\sum_{i \neq 2} \eta_{2 i}\right) N_{2} & \ldots & \ldots \\
\ldots & \ldots & \ldots & \ldots \\
-\eta_{k k} N_{1} & \ldots & \ldots & \left(\eta_{k}+\sum_{i \neq k} \eta_{i j}\right) N_{k}
\end{array}\right]\left[\begin{array}{c}
\frac{E_{1}}{N_{1}} \\
\ldots \\
\ldots \\
E_{k} \\
N_{k}
\end{array}\right]=\left[\begin{array}{c}
P_{i, 1} \\
\ldots \\
\ldots \\
P_{i, k}
\end{array}\right]
$$

where $\omega$ is the mid-band frequency, $\eta_{k}$ is the damping loss factor (DLF) of the subsystem, $\eta_{k i}$ is the coupling loss factor (CLF) among subsystems, $n$ is the modal density, and 
$P$ is the input power of the subsystem load.

For increasingly complex structures, their DLF values can be tested with the method of steady energy flow[10]. The key to this method is to measure the input power $P_{i n}$ of the excitation source in the system and the system energy $E$. The input power of the subsystem is

$$
\begin{aligned}
P_{i n} & =R_{f v}(\tau=0)=\int_{-\infty}^{\infty} S_{f v}(\omega) d \omega \\
& =\int_{-\infty}^{\infty} G_{f v}(\omega) d \omega=\langle f(t) v(t)\rangle
\end{aligned}
$$

The energy of the subsystem can be expressed as

$$
E=M\left\langle v^{2}(t)\right\rangle
$$

where $S_{f v}(\omega)$ is the cross-spectrum density of the bilateral force $f(t)$ and speed $v(t)$ at the excitation point and $G_{f v}(\omega)$ is the unilateral cross-spectrum density. According to the definition of the DLF, we can obtain the following from Formulas (2) and (3):

$$
\eta=\frac{P_{i n}}{\omega E}
$$

As for a three-dimensional space with volume $V$, superficial area $A$, and total side length $l$, the formula of its modal density is

$$
n(f)=\frac{4 \pi^{2} f^{2} V}{c^{3}}+\frac{\pi f A}{2 c^{2}}+\frac{l}{8 c}
$$

For the uniform rectangle plate, the modal density formula of the flexural vibration is

$$
n(f)=\frac{A_{p}}{2 R c_{l}}
$$

where $A_{P}$ is the area of the plate, $R$ is the turning radius of the section, $C_{l}$ is the longitudinal wave velocity in the material, and $c_{l}=\sqrt{E / \rho}$, where $E$ denotes the elasticity modulus and $\rho$ denotes the mass density.

The radiation efficiency of the two-dimensional plate is

$$
\sigma_{\text {rad }}=\frac{P_{r a d}}{\rho_{o} c_{o} A_{p}\left\langle v_{n}^{2}\right\rangle}
$$

In Formula (7), $P_{\text {rad }}$ is the radiated sound power, and $\left\langle v_{n}^{2}\right\rangle$ is the mean square velocity on the plate surface. Here, both the radiated sound power and the mean square velocity can be calculated with the finite element model and experimental measurement.

As for plates with special structures, such as stiffened plates, their modal densities cannot be calculated with the formula for uniform flat structures. Such calculation would result in large errors and necessitate extensive corrections. If a complex plate structure is substituted with a simple two-dimensional plate structure, then the calculation is simplified. The equivalent bending stiffness of a stiffened plate is defined as $B_{e q}$, and that of an ordinary plate is $B$. The ratio of the equivalent bending stiffness of the stiffened plate to that of an ordinary two-dimensional flat plate is defined as the stiffness multiplier $\alpha$, which is written as follows:

$$
\begin{gathered}
\alpha=\frac{B_{e q}}{B}=\left(\frac{n(f)}{n_{e q}(f)}\right)^{2} \\
B=\frac{E h^{3}}{12\left(1-v^{2}\right)}
\end{gathered}
$$

where $n(\omega)$ is the modal density of the structure, $B$ is the bending stiffness of the plate, $v$ is the Poisson's ratio of material, and $h$ is the thickness of the plate. The elasticity modulus of a stiffened plate structure for SEA can be determined by multiplying the elasticity modulus of two-dimensional flat materials with a stiffness multiplier. The equivalent stiffness can then be corrected.

\section{EXAMPLE OF NUMERICAL SimUlation ANALYSIS}

\section{A. Model of Cockpit floor of Fighter Aircraft}

We establish a cockpit floor of a fighter aircraft as Figure I shows. The spatial distribution of the stiffeners on the base plate is extremely complicated, their thickness is not uniform, and a corresponding subsystem based on standard SEA subsystems is lacking. In this example, we use the stiffness multiplier method to determine the equivalent SEA subsystem for the base plate.

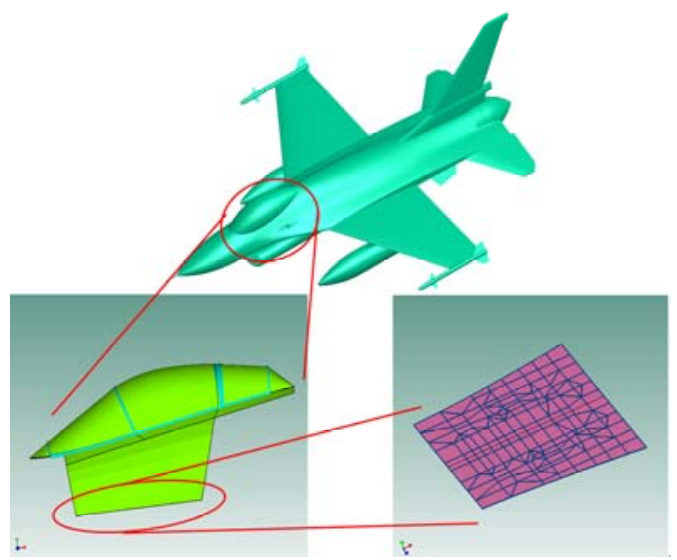

FIGURE I. COCKPIT FLOOR OF FIGHTER AIRCRAFT 
We combine the finite element method with the stiffness multiplier to modify the modal density. First, a detailed finite element model of the stiffened plate substructure must be established. Then, the calculation is conducted, including the calculation of the modal number within the frequency band, inherent frequency, and mode shape of the finite element subsystem. Subsequently, the equivalent SEA subsystem is established. At this point, a standard SEA subsystem is defined, and the modal number within the frequency band of the defined standard SEA subsystem is obtained with the VA One software. Then, the ratio of the modal number within the frequency band of the standard SEA subsystem and that of the finite element subsystem are defined as the stiffness multipliers. The material attributes of the SEA subsystem are modified, and the equivalent SEA subsystem is finally established. For acoustic problems, the radiation efficiency and transfer loss of the SEA subsystem can be used to (a) calculate the radiation efficiency of the SEA subsystem after correcting material attributes and (b) determine the radiation efficiency from the calculation of the finite element subsystem. Consequently, the CLF of the equivalent SEA subsystem can be modified.

The finite element subsystem model of the floor can be created with the VA One software, as shown in Figure II. The finite element subsystem model comprises 591 nodes and 584 units (including shell and beam elements). The sound fields on both sides of the subsystem are created with the SIF function of the VA One SEA software. The attributes of the structure are simplified to uniform material, thickness, and stiffened section characteristics (which are similar to those of the base plate materials on the floor). The thickness of the plate is $0.0015 \mathrm{~m}$, the material density is $2757 \mathrm{~kg} / \mathrm{m} 3$, the elasticity modulus $70 \mathrm{GPa}$, and the Poisson's ratio is 0.3 . The moment of inertia of an area of the beam sections are Ixx 8.004e-8 m4, Iyy 4.169e-008 m4, Jzz 1.273e-007 m4, and Qzz 3.726e-010 $\mathrm{m} 4$, the area is $0.000272 \mathrm{~m} 2$, and the perimeter is $0.256 \mathrm{~m}$.

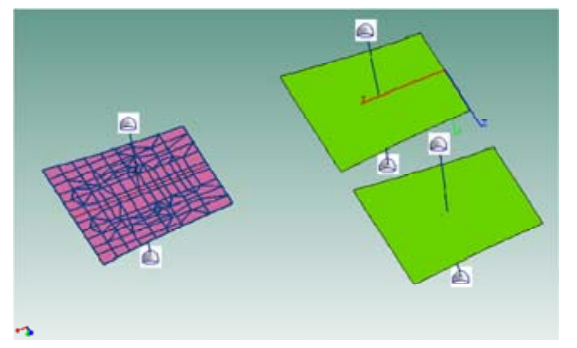

FIGURE II. MODEL OF THE VAONE SUBSYSTEM.

The material and thickness of the non-stiffened SEA plate subsystem (uniformed SEA) created with the VA One software are identical to those of the finite element subsystem. The sound fields on both sides of the plate subsystem are created with the SIF function as well. The analysis frequency domain is set at 1/3 OCT and $125-1,250 \mathrm{~Hz}$. The VA One built-in finite element solver is adopted to calculate the modal number of the frequency bands of the finite element, which should then be compared with that of the uniformed SEA subsystem, as shown in Figure Ш (A). Subsequently, the stiffness multipliers related to the frequencies can be obtained with Formula (1), as shown in Figure Ш (B). Finally, the mean of the stiffness multipliers related to the frequencies is used to modify the stiffness multiplier of the SEA subsystem.

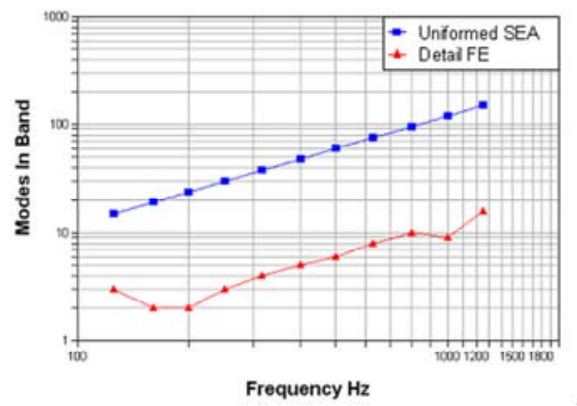

(A)

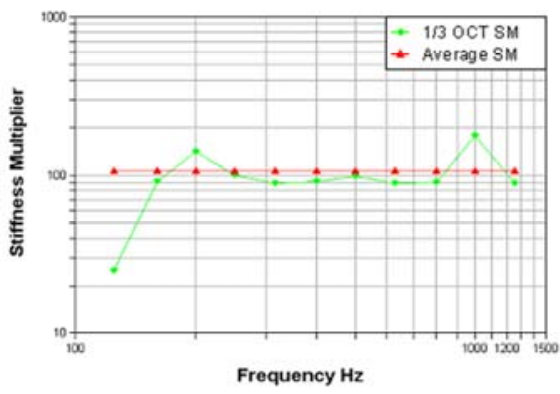

(B)

FIGURE III (A) MODAL NUMBER OF FREQUENCY BANDS AND COMPARISON BETWEEN FINITE ELEMENTS AND SEA; (B) MEANS OF STIFFNESS MULTIPLIERS AND FREQUENCY CHANGES AND DOMAINS

The average stiffness multiplier is applied to modify the uniformed SEA subsystem and finally obtain the updated SEA. The subsystem model is then created with VA One (Figure II).

\section{B. Comparative analysis of results}

The equations are an exception to the prescribed specifications of this template. You will need to determine whether or not your equation should be typed using either the Times New Roman or the Symbol font (please no other font). To create multileveled equations, it may be necessary to treat the equation as a graphic and insert it into the text after your paper is styled.

Figure IV (A) shows 20 decoupling concentrated force excitations imposed on the finite element system and 4 corner points imposed with simple support restraints. Eighteen sensors are imposed uniformly on the finite element subsystem to obtain the dynamic response at the response position. The dynamic response, radiation efficiency, and radiation sound pressure level of the structure can be calculated with the hybrid finite element/SEA in the VA One software (Figure V). Equivalent excitations are imposed on two SEA subsystems (uniformed and updated SEA). The spatial average dynamic response, radiation efficiency, and radiation sound pressure level of the structure can then be obtained (Figure V). 


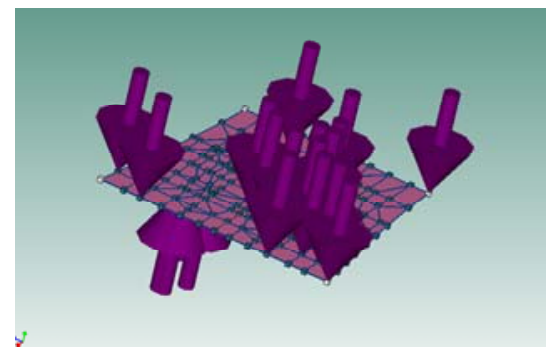

(A)

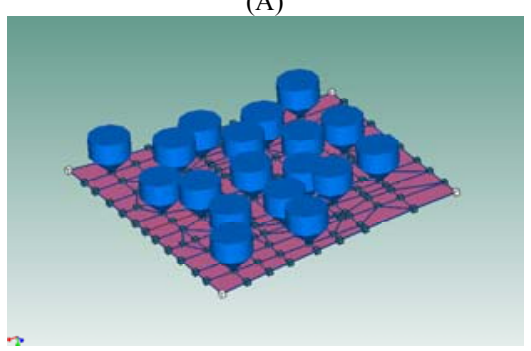

(B)

FIGURE IV. (A) MODAL PLING CONCENTRATED FORCE; (B) VIRTUAL SENSOR AT A RANDOM LOCATION

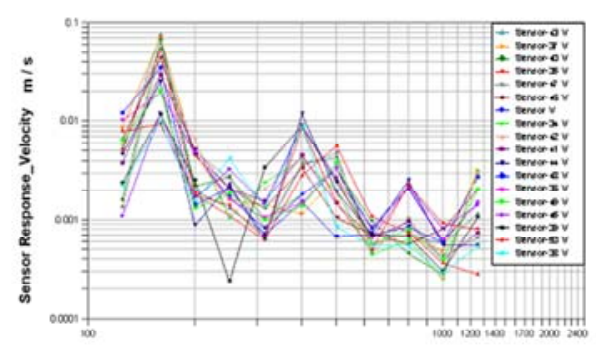

(A)

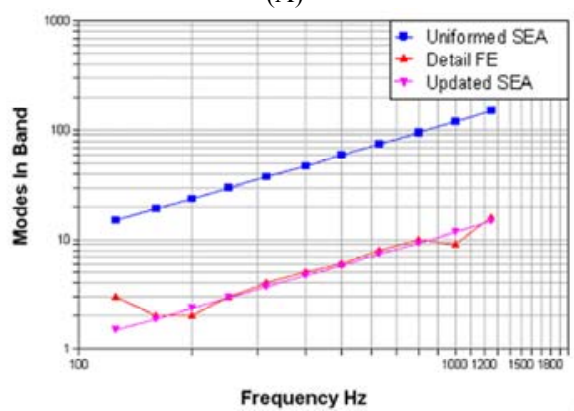

(B)

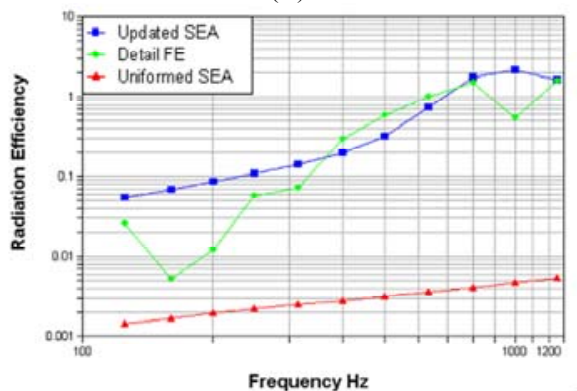

(C)

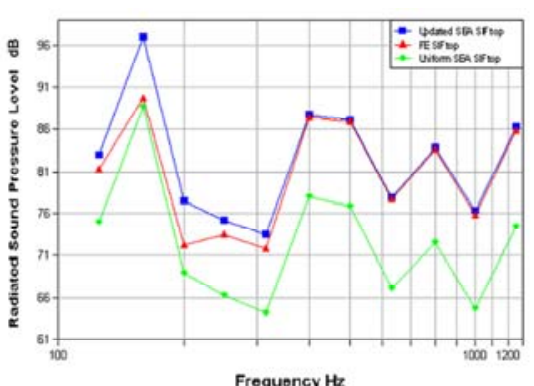

(D)

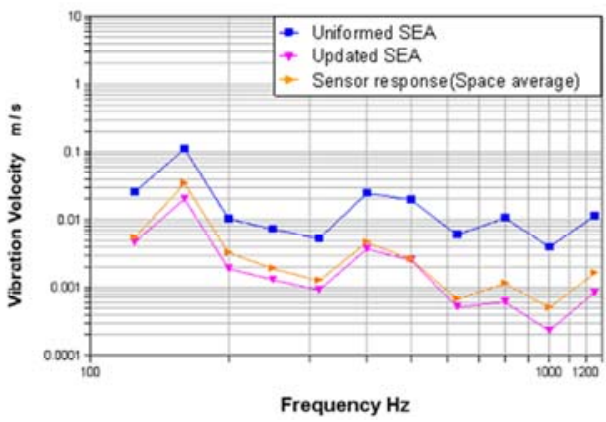

(E)

FIGURE V. (A) SENSOR RESPONSE OF THE FINITE ELEMENT SUBSYSTEM; (B) COMPARISON OF THE MODAL NUMBERS OF THE FREQUENCY BANDS OF THE FINITE ELEMENT AND UPDATED SEA; (C) COMPARISON OF THE RADIATION EFFICIENCIES OF THE FINITE ELEMENT AND UPDATED SEA; (D) COMPARISON OF THE RADIATION SOUND PRESSURE LEVELS OF THE FINITE ELEMENT AND UPDATED SEA; AND (E) COMPARISON OF THE SPATIAL AVERAGE DYNAMIC RESPONSES OF THE FINITE ELEMENT AND UPDATED SEA (THE SPATIAL AVERAGE DYNAMIC RESPONSE OF THE FINITE ELEMENT CAN BE OBTAINED WITH THE DYNAMIC RESPONSES OF EACH SENSOR)

Figure $\mathrm{V}$ indicates that the modal number of the updated SEA plate subsystem fits perfectly with that of the finite element subsystem. This result is a good reflection of the dynamic characteristics of the stiffened plate structure. The radiation efficiencies of these two subsystems are consistent. The low radiation efficiency at $1,000 \mathrm{~Hz}$ is caused by the compression of the plate structure. The difference in the radiation efficiencies at $150 \mathrm{~Hz}$ reflects the complex radiation characteristics at a coincidence frequency. Under this coincidence frequency, the SEA is mainly corrected with the stiffeners, irregular regions, or boundary radiations, which cannot fully reflect the characteristics of stiffeners.

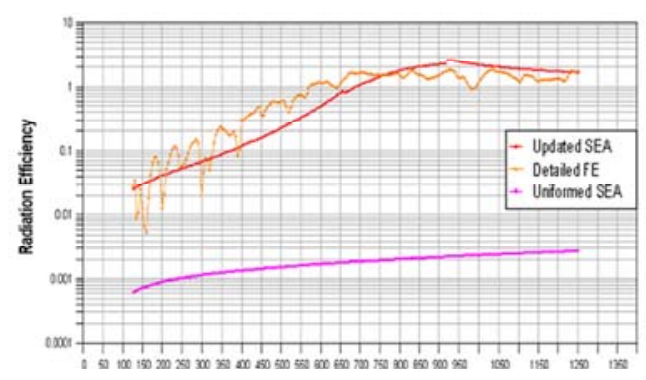

Frequency $\mathrm{Hz}$

FIGURE VI. RADIATION FREQUENCIES (EQUAL BANDWIDTH, $5 \mathrm{HZ})$ 
Figure VI shows the comparison of the equal bandwidth radiation efficiencies (bandwidth at $5 \mathrm{~Hz}$ ) and indicates changes in the low frequency radiation efficiencies. The differences in the radiation efficiencies are reflected in the radiated sound pressures. The prediction of the radiation sound pressure level of the updated SEA plate subsystem is consistent with that of the finite element subsystem. The differences in the radiation frequencies at $150 \mathrm{~Hz}$ reach nearly $6 \mathrm{~dB}$. The dynamic responses of the structure in Figure $V$ (e) show a good fit and an obvious correlation. The small difference can be eliminated through the well-distributed arrangement of sensors.

\section{CONCLUSIONS}

This study introduces the principle and application of correcting the attributes of the SEA subsystem with the stiffness multiplier method. Taking the cockpit floor of a certain type of fighter aircraft as an example, the SEA subsystem of the floor (updated SEA subsystem) is created with the stiffness multiplier method. Numerical verification is conducted on the stiffness multiplier with the hybrid finite element/SEA method of the VA One software. Through the comparative analysis of the calculation results, the following conclusions can be drawn. First, the SEA subsystem corrected with the stiffness multiplier method can effectively reflect the dynamic characteristics of the complex stiffened plate. Second, the radiation noise and vibration response of the structure analyzed with the updated SEA subsystem fit well with those analyzed with the finite element method. Third, the stiffness multiplier method can be used to create a SEA subsystem, which is similar to a complex stiffened structure. In this study, the transmission loss of the updated SEA subsystem is not explored but will be studied in future research.

\section{ACKNOWLEDGMENT}

This work was financially supported by the National Natural Science Foundation of China (Grant .No. 11404205) and the Fundamental Research Funds for the Central Universities of Ministry of Education of China (Grant No. GK201402012).

\section{REFERENCES}

[1] X. Chen, D. Wang, Z. Ma, "Simulation on a car interior aerodynamic noise control based on statistical energy analysis", Chin. J. Mech. Eng. vol.25, pp. 1016-1021, 2012

[2] Cristina Díaz-Cereceda, Jordi Poblet-Puig, "Antonio Rodríguez-Ferran Automatic subsystem identification in statistical energy analysis", Mechanical Systems and Signal Processing. vol. 54-55, pp. 182-194, 2015 .

[3] H. Yan, A. Parrett, W. Nack. "Statistical energy analysis loss by finite elements for middle frequency vibration". Finite Elements in Analysis and Design. vol. 35, pp. 297-304, 2000.

[4] M. Kassem, C. Soize, L. Gagliardini, "Structural partitioning of complex structures in the medium-frequency range An application to an automotive vehicle", J. Sound Vib, vol. 330, pp. 937-946, 2011.

[5] R. Lyon, Statistical Energy Analysis of Dynamical Systems, MIT Press, Cambridge, Massachusetts, 1975.

[6] V. Cotoni, R.S. Langley, P.J. Shorter, "A statistical energy analysis subsystem formulation using finite element and periodic structure theory", J. Sound Vib, vol. 318, pp.1077-1108, 2008.
[7] T. Onsay, A. Akanda, G. Goetchius, "Vibro-Acoustic behavior of bead-stiffened flat panels: FEA, SEA and experimental analysis", Proc. SAE Noise and Vibration conference, 1999.

[8] B. R. Mace, P. J. Shorter, Energy Flow Models From Finite Element Analysis. J. Sound Vib, vol. 233, pp. 369-389, 2000.

[9] R.H. Lyon and R.G.DeJong, Theory and Application of Statistical Energy Analysis, Butterworth-Heinemann, Newton, 1995, pp. 130-170.

[10] B. Mace, "Statistical energy analysis: coupling loss factors, indirect coupling and system modes", J. Sound Vib, vol. 279, pp. 141-170, 2005. 